\title{
Wolfgang Müller Entstehung von Recht und Staat und frühes griechisches Recht
}

\section{Einleitung}

Der Autsatz von Wesel ist entstanden aus einem Abschnirt seiner Vorlesung zum römischen Rechr, der sich mit der aFrühgeschichte des Reches * befaßc, seiner Frühgeschichte nämlich in der Ưbergangszeit zwischen Genuilgesellschaften und Protostaaten; Belege zieht er vor allem aus der ethnologischen Literacur. Uber die Entsrehung des römischen Rechts und erst recht über die des Rechis unserer bürgerlichen Gesellschaft sagt Wesel in diesem Aufsacz nichss (ich kann aus eigener Kenntnis auch nicht einschätzen, was er in seiner Vorlesung dazu gesagt hat). Die - vermurlich von Wesel niche beabsichtigte - Wirkung seines Aufsatzes auf mich und wohl auch manchen anderen Leser geht allerdings über dessen eigencliches Thema hinaus. Gerade in der akruellen politischen Situation und ihren divergenten Einschätzungen in der Linken könnte der Aufsatz eine Lesarn stützen, in der Freiheit/Gleichheit/Brüderlichkeit in frühen Gesellschaften und Unterdrückung/ Herrschaft/Staat in Industriegesellschaften einander dualisciscb gegenübergestellt werden. Durch die thematische Einschränkung des Themas auf die Entstehung von Recht in frühen Gesellschaften können m. E. Tendenzen gefördert werden, welche aus fernen Gesellschaftsformen bestimme als posiciv eingeschätzte Aspekte unvermittelt übertragen wollen. Solche unreflekrierten Ubbertragungsversuche können zu einer antihistorischen Weltfluche führen (bei aller Anerkennung der Fruchtbarkeit des Verfahrens, unsere Krisengesellschaft mit den Lösungsformen anderer Gesellschaften für grundlegende Probleme menschlicher Lebenserhaltung kritisch zu konfroncieren).

Diese von mir vermuteten Wirkungsmöglichkeiten von Wesels Aufsatz hätten durch entsprechende Hinweise zumindest erschwert werden können. Der Sache nach scheint mir das Missing Link der Weselschen Argumentarion in der Darstellung der weireren Stufen der Entwicklung von Recht und Staac zu den für die moderne bürgerliche Gesellschaft charakreriscischen Formen zu liegen. Die wichtigste Zwischenstufe ist nach meiner Auffassung die Herausbildung des griechischen und besonders des römischen Rechts; zunächst weil es Rechtsformen von in vieler Beziehung bereits bürgerlichen, von Warenverhältnissen deurlich bestimmten Gesellschaften sind; sodann weil speziell das römische Reche bei der Herausbildung rechtlich vermittelter staatlicher Herrschaft im modernen Europa eine fördernde Rolle gespielt hat.

Mein vorliegender Beitrag versucht im Hauptreil, die eben bezeichnete Lücke an einem Punkt exemplarisch zu schließen, am Fall der Entstehung des griechischen Rechrs. (Vermutlich besser wäre das am römischen Rechc zu zeigen gewesen, und sicher viel besser als ich wäre dazu Uwe Wesel in der Lage). Durch die Interpretation einiger elementarer Kategorien, wie der Person und ihrer Auconomie sowie des Eigentums und des Vertrags, soll die Beziehung dieses Rechts auf eine Gesellschaft gezeigt werden, welche durch die Ausbreitung des Warentaustauschs und damit 
zusammenhängender abstrakter Beziehungen bestimmt ist und welche als prinzipiell neuartiger Epochalryp zu begreifen ist, im Unterschied zu früberen Typen. Nach diesem Haupteil versuche ich, die oben angedeutete dualistische Lesart anhand einzelner Argumente aus Wesels Aufsarz kritisch zu beleuchten. Mein Hauptargument gegen eine dualistische Betrachtungsweise liegt aber nicht in der Erörterung einzelner Topoi, sondern in der Darstellung von solchen historischen Vermirtlungsschritten, welche in vieler Beziehung für das Verstàndnis und die Kritik der bürgerlichen Gesellschaft und des auf sie bezogenen Scaares näherliegend sind. Was Recht und Staas in der bürgerlichen Gesellschaft bedeuten, vor aljem: welche grundlegenden Ambivalenzen und Widersprüche sie auch in ibren einzelnen Kategorien und Formen enthalten, das kann man $m$. E. recht gut an deren Herausbildung im Rahmen der griechischen Polis lernen. - Um Mißverständnisse auszuschließen, stelle ich explizit fest, daß ich mich im vorliegenden Aufsatz nicht mit Uwe Wesels Beitrag als solchem auseinandersetze, sondern dessen mögliche Wirkungen in der gegenwärtigen Situation antizipiere, daher eine auf die gegenwärtige Gesellscbaft vermittelnd bezogene Ergänzung vorlege und nur am Rande die von mir vermuseren Wirkungen anhand Weselscher Argumence kritisiere.

\section{Warenaustausch, Recht, Abstraktion}

Meine folgende Erönenung gehr davon aus, daß das Charakteriscische des bürgerlichen Rechts an dessen Anfängen verständlich gemacht werden kann und daß diese Anfänge für den 'Epochalryp 'Bürgerliche Gesellschaft in der griechischen und der römischen Anuke festzumachen sind (was keineswegs die Vorsrellung einer historischen Kontinuität impliziert). Wesentliche Momente (genauer kann das hier nicht ausgefübre werden) dieses Gesellschattstyps sind Warenaustausch und abstraktes Recht. Die Fähigkeit und die Norwendigkeit der Abstrakcion, der Arbeit der Abstrattion, die Ausbreitung der Abstraktion auf immer weitere Lebensbereiche unterscheidet diesen Typ von anderen (bisher nur früheren). Gerade an der Erörterung sfrühbürgerlicher Rechtskategorien kann man den spezifischen Charakter jener vom Juristen alleäglich und selbstverständlicb geleistecen Abstraktionsarbeit ins Bewrißssein und aus der Selbsiverseändlichkeic herausheben. Zugleich kann deudich werden, wie sehr die Fähigkeir zur Abstrakcion nur als Variance anderer Formen der Abstraktion zu verstehen ist.

Ordnung, Disziplin, Regellhaftigkeit sind immer als besondere Charakterisrika der juristischen Tätigkeit erschienen; sie setzt konkret Ungleichartiges gleich, ssubsumiertr, verändert damit und macht beherrschbar. Aber gerade diese rücksichtslose Gleichgültigkeit gegenüber dem Einzelnen und Besonderen, diese Kraft der ,Abstrakrion, des , Abstrahierense vom sinnlich Konkreten, ist Kennzeichen wissenschaftlicher Arbeit überhaupt. Diese Gemeinsamkeit wird vielleicht erst heute wirklich deurbich, wo jene rücksichtslose Gleichgültigkeit gegenüber dem Besonderen - letzten Endes gegenüber der inneren und äußeren Natur - als technologisch, d. h. als wissenschaftlich-technisch gelenkte Produktion übermächtig wirksam wird. Der Subsumtionstärigkeit der Juristen gegenüber har sich immer ein Rest vernünftiger Kritik erhalten ('Fiat justitia, et pereat mundus!c). Das Gerneinsame der verschiedenen Formen der Abstraktionsarbeit wird aber schon in den Epochen ihrer Herausbildung rechr deutlich - besonders bei der Entscehung wissenschaftlicher Rationalizäc in der griechischen Anrike.' Das fällt wohl am stärksten bei der

I In meiner Uncersuchung: Geld und Gcist (Zur Entsehungsgeschichte vou Identitätsbeorußstsein und Racionalieär seit der Antike. FrankfuruNew York 1977) habe ich diese qualitaive Neuarigkeit 
Herausbildung einer erstmals wirklich abstrakten Machemarik (als Geometrie) ins Auge. Die meisten Mathematikhistoriker sind sich darin einig, daß erst in der griechischen Mathematik Wissen um seiner selbst willen gesucht und begründer . worden ist; Scout spricht vom "Begriff eines im strengen Sinn deduzierenden Beweises, der Methode, den Gegenstand durch eine geordnete Abfolge von Theoremen zu entwickeln, die auf Definitionen, Axiomen und Postulacen beruhen, und der fortgesetzten Bemühungen um vollständige Verallgemeinerung und Abstrakcion. $\kappa^{2}$ Entsprechendes gilt für die zuerst von Aristoceles zusammenfassend ausformulierte Logik. Die uns so selbstverständliche reine Erkennenisbeziehung, die Fähigkeit zur abstrakten Rasionalicät des mir anderen in dieser Beziehung identischen Erkenntnissubjekts, nicht weniger aber auch die Existenz einer Welt identischer und in ihren Beziehungen durch Gesetze bestimmbarer Objekce ist ein allgemeiner Ausdruck jener neuartigen Beziehung der Menschen zu ihrer Umwelt. Diese bei allen Differenzen' doch erstaunlich hohe strukturelle Ubereinstimmung ist es, die uns möglich macht, literarische und wissenschaftliche Schriften der griechischen und römischen Antike fast so zu lesen, als ob sie aus unserer Welt stammten. Ein Aristoteles oder ein Cäsar steht vernutlich gerade dem Wissenschaftler und nicht zuletzr dem Juristen näher als ein - bürgerlich - Unggebildeter der zeitgenössischen Gesellschaft; den ,Elaboraced Code lernt man immer noch am besten an der laceinischen Hochund Herrschaftssprache. - Es isc hier nicht der Ort, den umfassenden Charakter der abstrakcen Rationalitär und ihrer Ausbildung mehr als mit diesen Andeutungen zu belegen. Entscheidend ist vor allem die Erkenntnis, daß abstrakte Rechtsbeziehungen zusammen mit anderen abstrakten Beziehungen in einem Umfeld umfassender gesellschaflicher Veränderungen zuerst entwickelt werden und daß durch ihren abstrakten Charakter diese Beziehungen der Menschen untereinander und zu ihrer naürlichen Umwelt etwas epochal Neuartiges darstellen. Mit Recht spricht Popper von einer der riefgreifendsten Revolurionen in der Geschichte der Menschbeit überhaupt. ${ }^{4}$

Wenn sich die folgenden Ausführungen auf die Kategorien der Person, des Eigentums und des Vertrags und ihre - begrenzte - Entfalcung im griechischen Recht

besonders anhand Inühgriechischer Literatur herauszuapbeiten versuche (vgl. dort III.B). In dieser Absichr habe ich die Frage nach den historischen Vorstufen wohl etwas zu sehr in den Hincergrund gedrünge. Es wäre erws genauer zu fragen, in welcher Weise der Vergleich der Opfergaben der

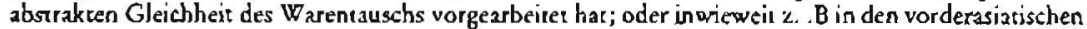
Staatsvenrägen bercits abstrakte Rechtskatcgorien auttreten. So nachthalog und folgenreich der ge sehicheliche Einschnit cinzuschätzen ist, der mit dem Namen Athens bezeichnet werden kann, so muß man sich doch methodisch von der Annahme eincr creatio ex mihilo (oder: e nummo) hüten: was war vor ders Anfang?

2 Ubersetzt aus J. F. Scom, A History of Machematics. From Anriquity to the Beginning of the 19th Century. London $1960^{\circ}$, S. 13. Weitere Hinwcise in meincr o. a Untersuchung, S. 373 f. J. Klister stelle kürzlich die These auf, daß Operationalismus (bzw. Konstrukeivismus) und Platonismus dic beiden grundlegenden wissenschalesiheoretischen Posicionen der exakten Wissenschafuen seien, und verfolgze deren Gegensatz für die Mathemavik bis auf die gricchischc Anvike zurück (Eudoxos vcrsus Placon) (Kliver. Operacionalismus. Kritik u. Geschichte einer Philosophie d. exakten W/issenschaíten. StutgarBad Cannstatt 1971).

3 Die Differenz aul der Ebene der Erkenninisbezichung ist bercits dadurch angedeutet, das über die griochische Naturwissenschaft nicht daseelbe wie uber die Machemaeik zu berichten ist. Wie die Produkrionsweise im ganzen wecnig weiterentwickelt worden ish lehle auch der aktive und speziell der durchs Experiment vermittelte Zugriff aut die Natur, welcher diese zu Reakuionen auf vorbesummten Bahnen veranlaBt; dic subjekrive Tätigkcit des Erkennens blieb in der Anrike primär Kontemplacion, Anábnelung un dic als übcrlegen, ewig, wabr verstandene Objektivität. Die Vernachlässigung dieser neven Dimersion, die man am ehesten mit dem Namen Galileis verbinden karn, ist ein Mangel der in der erstcn Aumcrkung crwähnten Untersuchung. Vgl. zur Krizik: L. Hieber, Sohn-Rethels Bedeutung für dic Selbsureflexion naturavisscnschaflicher Arbeit. in: Prokla. Zeitschr. I. pol. Okonomie u. sozialist. Politik, Nr. 29 (1977), S. 77-99; H. König, Tauschabseraktion und Geschichtc. Überlcgungen zu R. W. Mülder, Geld u. Geist. In: Leviachan. Zeiuschr. I. Sozialviss. Nr. 1/r978, S. 1 27-1 50.

$\left.{ }_{4} V_{g}\right)$ K. R. Popper, The Open Socicry and its Enemies (1945). London 1966, Bd. 1, Kapitel ro. 
konzentrieren, so ist damit also nur ein Moment einer ganz umfassenden Entwicklung herausgehoben. Und das gilk auch im engeren Sinne, für die Sphäre des Rechrs: wenn man die Kategorie der Person untersucht, so kann man dies nichr, ohne zugleich weirere, damit unmittelbar verknüpfte Kategorien zu berühren. (Ich nenne einige von ihnen, um diesen Umkreis zu bezeichnen: Rechtssubjekt, Privateigentümer, Individuum und dessen Freihein Auronomie und Gleichheit, Willen und Willensfreiheir, Eigentum und Freiheit des Eigentums, Vertrag und Vertragsfreiheit, Vertragszwang, Haftung, Rechtsetzung, allgemeines Gesetz, Rechrsauslegung, Schuld, Strafe, Zwangsgewalt, Monopol physischer Gewaltanwendung, Souveränitär, Staat.)

\section{Entstehung und Bedeutung des Warenaustausches in der Polis}

Die Ausdehnung regelmäßiger Warencauschbeziehungen ist geknüpft an die Anerkennung der Freiheit der Tauschreilnehmer, der Freiheit der Person; zumindesr historisch sind beide Momente eines einzigen Prozesses. Zugleich mit der Entwicklung des Warenaustauschs sind in Griechenland und vor allem in Achen in der Epoche zwischen dem 7. und dem 4. Jahrhundert Realieät und Begriff der Person herausgearbeitet worden; das späte 7. und das 6. Jahrhundert ragten nicht zu Unrecht durch die Namen der großen Gesetzgeber Drakon, Solon und Kleisthenes heraus. Im folgenden soll zunächst die Entwicklung des Warenaustauschs und der Warenproduktion in jener Epoche knapp skizziert werden. Danach soll am Begriff der Person und ihrer strafrechelichen Veranrwortlichkeit das Neuartige und Vorwärtsweisende, darn aber an den Begriffen des Eigentums und des Vertrags die charakteriscische Beschränktheit jener Entwicklung, selbst im Fortgang der späteren Jahrhunderte, dargestellt werden.

Der Großceil der materiellen gesellschaftlichen Arbeir wurde in Athen im 6. Jahrhundert (und prinzipiell auch noch in den folgenden Jahrhunderten) für die Selbstversorgung in der land- und hauswirtschaftlichen Produktion verausgabr, vielfach, wenn damals auch sicher noch nichr überwiegend, als unfreie Arbeit innerhaltb des Oikos, der mehr oder weniger großen ,Hauswirtschafu (die zugleich Lebensgemeinschaft in einem umfassenden Sinn war). Nur ein geringer, allerdings schnell steigender Teil der Produkte ging in den Auscausch ein (der wegen des technischen Entwicklungsstands der Transportmittel in der Antike vorherrschend Seehandel war). Vieles davon verdient freilich den Namen Handel noch nicht; erst recht sind Vorstellungen einer sich durchsezzenden Industrier oder gar ,fabrikmäßigen Industrier auch für Achen durchaus febl am Platz, obwohl solche Begriffe in der Literatur immer noch verbreiter sind. Immerhin muß die Herscellung von keramischer Exportware in Handwerksbetrieben jedenfalls in der 2. Hälfre des 6. Jahrhunderts stark zugenommen haben, wie die Funde in der Ăgäis und in Süditalien zeigen. In diesen Töpfererzeugnissen wurden attisches Olivenöl und Wein exportiert; Importwaren waren vor allem Getreide, Metalle (außer Silber), auch wohl schon Sklaven. In der Landwirtschaft entwickelt sicb bereits eine über den Warenaustausch vermitreite Arbeitsceilung (Olbäume statt Getreide). ${ }^{6}$ Athen war spärestens in der 2. Hälfte des 6. Jahrhunderts ein Handelszentrum (nichr eine Handelssradt),

Sie Iolgenden drei Abschnitre sind ein Auszug aus meinem Aufsaz: Momente des büngerlichen Staates in der griechischen Polis. In: Probleme des Klassenkampfs. Zeisschrift für pol. Okonomie u. sozialist. Policik. Nr. $17 / 18$ (1975), S. 1-25. Verschiedene Passagen sind dort ausführlicher und mil genaueren Nachweisen; einzeloc Formulierungen sind für die vorliegende Fassung veränder worden.

6 Vgl. Genaucres in meiner Untersuchung: Geld u. Geist, 2. 2. O., S. 11s ff. 
das die erwas äteren Handelszentren am Saronischen Golf (Aígina, Korinch) zu überflügeln begann. Ein rasch zunehmender (insgesamt aber doch recht kleiner) Teil der landwirtschaftlichen und vor allem gewerblichen Produkcion war für den Export bestimmt.

Ein besonders eindrucksvolles Indiz für den Umschwung im 6. Jahrhundert bietet die Geschichce der Münzen. Nach der Prägung der ersten Münzen aus Elektron in Städren an der kleinasiacischen Küste gegen Ende des 7. Jahrhunderts finden sich die ersten Münzen aus Silber in Aígina, der Nachbar- und Konkurrenzscadt Áchens. Man kann davon ausgehen, daß diese Münzen auch in Achen umgelaufen sind. Eigene Münzen prägt Athen erst gegen Mirte des 6. Jahrhunderts; erhebliche Verbreitung findet das achenische Geld erst in der zweiten Jahrhunderthälfte. Um so beginnt in Achen die Prägung jener berühmten, ,Eulen genannten Silbermünzen, die die anderen Währungen bald aus dem Fernhandel zurückdrängten und für Jahrhunderte die Rolle eines Weltgeldes des Mittelmeergebieres spielen sollten. Schon vorher hatte auch die Prägung von kleineren Denominationen begonnen, die allmählich immer mehr unterteilt wurden und die für die Verwendung des Münzgeldes im alleäglichen Detailhandel Voraussetzung sind, wie er für das s. Jahrhundert auch literarisch bezeugt ist. Gerade für diese Kleinmünzen war der Schria zur Silberprägung norwendig; materielle Bedingung waren die reichen attischen Silbervorkommen. (Neben dem durch Münzgeld vermittelten Austausch hat aber sicher immer auch unmittelbarer Tauschhandel scattgefunden.) Die Eulen-Silbermünzen werden als bewußter und erfolgreicher Versuch der athenischen Tyrannen verstanden, der eigenen Währung die Rolle des allgemeinen Äquivalents im Fernhandel zu verschaffen. Thre äußere Gestals weist selbst deutlich genug auf die sie garancierende Staarsmacht hin, die sich gegen nivalisierende Adelsgeschlechter durchgeserzt hat. Denn waren die vorausgehenden Silbermünzen mit den verschiedenen W/appen dieser Geschlechter geprägt worden, so haben die Eulenmünzen schon die bis heute übliche Gestalt von Münzen: das Profil der Stadtgötcin und , Landesmutter< Athēnē auf der Vorseite, die ihr heilige Eule als Wappentier zusammen mit den Initialen ATHE auf der Rückseite.?

Noch vor den Tyrannen trit der berühmte Solon auf, der sonderbevollmächeigte Jahresbeamte des Jahres $594 / 93$ und nach Auffassung vieler Autoren der erste Staatsmann der europäischen Geschichter. Diesen Titel hat man ihm vor allem wegen seiner Täugkeit als Schlichter der Klassenkämpfe zwischen Großgtundbesitzern und verschulderen, ja teilweise versklaveen Kleinbauern verliehen. Indem er die Kleinbauern durch einen allgemeinen Schuldenerlaß befreite, legte er eine wichrige Basis für die Ausdehnung von Handel und Gewerbe und sicherte zugleich die Grundherren vor einer radikalen Landaufteilung bzw. offenem Bürgerkrieg. In unserem Zusammenhang interessier besonders die dem Solon zugeschriebene Reform von Maß, Gewicht und Münze. Während seine bisher angenommene Rolle bei der Münzreform neuerdings vorwiegend als nachträgliche Zuschreibung im 4. Jahrhundert verstanden wird, bezweifelt man nicht, daß er Gewicht - und Maßsystem neu geregelt hat, und zwar mit der Absichs, Achen an ein für den Handel aussichtsreicheres System anzuschließen und die Abhängigkeit vom System der Konkurrenzscadt Aigina zu beseirigen. Während die Forschung sich aber in größter Ausführlichkeit mit der Interpretation der Uberlieferung der solonischen Reformen beschäfrigt, wird die Tatsache der einheitlichen Regelung der Maße selbst kaum grundsätzlich gervürdigt. Es wird nicht gesehen, daß eine derartige Regelung überhaupt nur in einem gesellschaftlichem Encwicklungsstand notwendig und sinn-

$7 \mathrm{Vgl}$. C. M. Krasy, The Archaic Owls of Athens. Classification and Chronology. In: Numismaric Chranicle. 6th Series, Bd. 16 (1956), S. 43-68. 
voll sein kann, wo die unmitcelbare, autoritative Verteilung der Produkte z. B. gemäß den Stanmestraditionen nicht mehr alkein herrscht, sondern wo ein Teil der Produkce regelmäßig in den Austausch eingehr, $\alpha$. h. in bescimmten Gebrauchswertproportionen gleichgesetzt wird, wo also prakcisch und unbewußt von konkreter Verschiedenheit abstrabiert wird.

Halten wir einstweilen fest: die Entwicklung des Warenaustauschs war in Athen am Anfang des 6. Jahrhunderss ausgedehnt genug, um eine einheidliche Regelung der Warenmaße und Warengewichte durch eine über die einzelnen Gruppen und Individuen der Gesellschaft erhobene staatliche Aucoricät zu bewirken; gegen Ende desselben Jahrhunderts war Athen als Handelszentrum enrwickelı genug, um einer eigenen, vom Staat cinheidlich geregelten und garantiersen Münze und Münzordnung zu bedürfen. (Beim Begriff `Staar muß man moderne Vorstellung zunächst \{ernhalten und nur das darunter verstehen, was tatsächlich eingeführt ist.) Daneben existieren im übrigen weirerhin vergleicbsweise sehr werig entwickelre Poleis und auch Stammesgesellschaften.

\section{Herausbildung der Freiheit der Person und der offentlichen Zwangsgewalt}

Es soll nun genauer die Herausbildung der Autonomie der Person und unmitcelbar damir zusammenhängend́ einer öffentlichen $Z$ wangsgewalt in dieser Zeit dargescellt werden, und zwar an der Entwicklung, die von der Selbsthilfe der Gruppe bzw. des Clans gegen Unrechrstärer (erwa durch die Blucrache) zur Herstellung einer öffentlichen Gerichtshoheit bej einer erheblichen Zahl von Delikten im 6. Jahrhundert führte. Manche unserer Darlegungen werden sich mit denen Wesels berïhren oder überschneiden; allerdings interpretieren wir diese Entwicklung, wie oben ausgeführ, im Zusammenhang der Ausbreitung des Warentauschs, fassen die Encwicklung des frühen Rechis also als Moment einer Gesameencrwicklung, die ohne die Entfaltung der Warenbeziehungen nicht möglich gewesen wäre, jedenfalls in der ciefgreifenden Ausprägung, die für die Verhältnisse Griechenlands und vor allem Athens charakieristisch und durch die Herausbildung der Abstraktionstätigkeit bestimmt ist.

Schon der moderne Begriff', Unrechestäter ist für die sogenannee vorgeschichtliche Zeit fehl am Placz; noch Odysseus kann in der Odyssee das Inkognito des vor der Blutrache fliehenden ,Mörders w wählen, ohne deswegen irgendeine Verfolgung oder Diskriminierung befürchten zu müssen, solange er sich nur außerhalb der Reichweite der berroffenen Gruppe aufhält. Nur die Gruppe tritt dem Verlerzten zur Seite, die überhaupt für seine gesamte Existenz die Grundlage isc, so vor allem die parriarchalische Großfamilie oder Hausgemeinschaft (Oikos), daneben auch der kleinbäuerliche Verband der Nachbarn oder die kriegerisch-ariscokratische Vereinigung der Kameraden (letztere beiden Gruppen ein Hinweis auf klassenspezifische Verbindungen). Die Rache kennt, im Unterschied zur Strafe, keinerlei allgemeine Schranken; ihr einziger Maßstab ist das Ehrgefühl der Becroffenen. Daher kann sie mit deren Einverständnis auch bei Mord durch eine Buße (,Wergelds) abgelöst werden. Eine wichtige Einschränkung der Selbschilfe liegt bereits in der erst freiwilligen, dann obligacorischen Anerkennung eines Schiedsspruchs, die Aufschub und möglicherweise eine Modifikation der Selbsthilfe bedingt. Der obligatorische Schiedsspruch ist ohne ein Minimum zentralisierter Autorität nicht möglich, die allerdings niche mehr sein mag als die Versammlung der Gemeinfreien oder der Adeligen. 
Eine "Art der Souveränität ${ }^{8}$ wird deutlicher, sobald der Schiedsspruch sich auf anerkannte und bekannte, Gesetze, stützt, in denen die Tatbestände benannt und die Bußen fixiert sind. Ein für das griechische Rechusbewußtsein entscheidender Schritt liegt in der schriftichen und öffentlichen Fuxierung solcher Geserze und in der Anerkennung der Gleichbeiz aller Vollbürger vor diesen Geseczen. Damit isc die Stufe der Kodifikationsbewegung des 7. Jahrhunderts erreicht, die in Achen mit den Namen Drakons und Solons bezeichnet ist und mit der die Geschichte des Rechtes und damit auch das Sraates in dem uns interessierenden Sinne beginnt. (Wenn einzelne ,Geseczgeber genannt werden, so ist davon auszugehen, daß sie im allgemeinen nur den erreichten Stand der Rechrsauffassung kodifiziert haben, nicht etwa neues Recht schöpfen.)

Die älteste Uberlieferung ist das Blutrecht des Drakon (gegen Ende des 7. Jahrhunderts). Nur nach gerichtlicher Klärung von Tatbestand und Willensrichtung des Totschlägers wird hier gegebenenfalls die Selbsthilfe zugelassen; es herrscht also Gericheszwang. Während in älzerer Zeit nur das Resulcat, der Tote, zählte (mit allen Begleiterscheinungen fortdauemder Blucrache, wie sie noch heute etwa aus Teilen Jugoslawiens bekannt sind), wird im drakonischen Gesecz die fundamentale Unterscheidung zwischen Mord und Torschlag einerseits und fahrlässiger Törung andererseits getroffen. Erkennt das Gericht auf vorsätzliche Törung, so wird der Tärer dem sofortigen Zugriff der Verwandten des Getöteten ausgeliefert. Wird auf unvorsätzliche Tötung erkannc, so wird zwar das Recht auf Selbschilfe bestätigt, jedoch mit der Maßgabe, daß der Verurzeilte freien Abzug erhält und daßs sein Eigentum nicht für Repressalien freigegeben wird. Außerdem kann auf bußlose Tötung erkannt werden, z. B. bei Gegenwehr gegen Raub; die Angehörigen verlieren dann ihren Anspruch auf Rache.

Das für die weicere Entwicklung Entscheidende isc die im Begriff des Vorsaczes enthaltene prinzipielle Anerkennung der Freiheit des Willens, der Autonomie und daber Verantwortichkeit des Einzelnen bei der Tat. Die Anerkennung dieser Autonomie bedarf allgemeiner, über den einzelnen Sippenverbänden und dergleichen stehender, öffentlicher Ausorität und Sankionsgewalt: es muß in allen Fällen mit dem Gerichtsurteil die Möglichkeit seiner Durchsetzung verbunden sein. Andererseits geht der staatliche Eingriff im schwersten Fall noch am wenigsten weit: die Selbsthilfe wird bei Mord nur vorübergehend aufgehalcen (die eigentliche Hinrichtung (durch eine Behörde) gehörc einer späteren Zeit an). Und nur die Blursverwandren können Anklage erheben; noch in 4. Jahrhundert kam es vor, daß Mord nicht strafrechtlich verfolgt werden konnte, weil der Erschlagene keine Angehörigen hatte. Das noch deudich erhaltene Vorstaatliche beim Blutrecht zeigt sich auch bein Recht auf Vergebung: Die Angehörigen konnren sich mit einem Sühnegeld begnügen, der Sterbende konnte dem Täter verzeihen und ibn damir vor Befleckung und daher Rache schützen."

Die noch nicht vollsëndig erreichre Verdrängung der sPrivarjusciz^ erklärt auch die sprichwörliche, drakonische Strenge` der smic Bluc geschriebenen، Geserze Dra-

$8 \mathrm{Vgl}$. L. Gernet, Uber dea Bugriff des Uneils im griechischen Recht. In: Zur griechischen Rechtsgeschichce. Hrsg. E. Bemeker. Darmstadt 1968, S. 374-412, hier S. 382 . . Wir crinnern dabei nur an die Wendungen von Aristoteles, für den das dikázein ein norwendiges Element der policischene Macht ist....e (cbd.).

9 Derariges ist bis herute im islamischen Rechesbercich üblich, z. B. in Saudi-Arabien; vgl. z. B. den Bericht im .Spiegcle, Nr. 33/1973, S. 58: "Nach dem koranischen Scharia-Recht wird dic Todessurafe nicht vollstrecks, wenn die geschädige Panci vergibt. Im November solltc in Dschidda ein junger Mann hingerichter werden, der einen Gleichaltrigen im Streit getöter hane. Der Henker hare schon zum Schlag ausgeholt. $D_{2}$ stieß die Mutzer des Toren unter ihrem Schleier hervor, daß nun kein Blut mehr fließen solle. Der Verureilie war daraufhin frei. Einc Ambulanz brachic ihn mit heulender Sirene in cines der modernen Krankenhäuser zur Behandlung seines Schocks.\& 
kons $^{10}$, auch bei anderen Delikten, z. B. Diebstahl: sie war Voraussetzung für eine Ablösung der Selbschilfe. Noch in shistorischere, d. h. schriftlich überlieferter Zeir kann in einigen Gebieren der naches überraschee Dieb mißhandelt, verwunder oder gerötet werdén, ganz wie es dem Geschädigten zumure ist. Enckommt der Dieb, wird er mit Hilfe der Nachbarn verfolgt; Hausbewohner müssen die Hausdurchsuchung zulassen. Entsprechendes gilr für die Abwehr bei Mordversuch, Raub und auch für Ehebruch, der als Eigentumsverleczung gilc. Gerade beim Ehebruch werden auch die ersten Versuche eines gesetzlichen Eingreifens gur sichtbar, das ja mir der Fesclegung eines Tarbestandes beginnen muß. Was wir mir verminderter Zurechnungsfähigkeit bezeichnen würden, wird in einem Gesetz von Tenedos in der Weise ausgedrückt, daß der Ehemann zur Törung die Axt benutzen müsse. Denn die Axt war das Werkzeug, das im Innern jedes Hauses sofort zur Hand war; andere Waffen mußten erst herbeigeholt werden, was den Vorsatz unterstellt. In die Zeit des Solon führt uns ein diesem zugeschriebenes Geserz, wonach das Racherecht nur bei der Ertappung in flagranti eintrat. Die Bestimmung des Tarbestands ist hier schon fortgeschrittener; denn wenn bei dem Gesetz von Tenedos das Merkmal ganz äußerlich aus der Art der Rache gewonnen ist, tritt bei dem artischen Geselz das Racherecht nur ein, wenn der Ehebrecher, Glied im Glied habends angetroffen wird."

Den Úbergang von der gesetzlichen Einschränkung des Tatbestands zur Anerkennung der staatlichen Gerichtshoheir läßs noch deutlich das Apagoge-Verfahren des athenischer Rechts erkennen. Ein Solon zugeschriebenes, vermullich älteres Gesetz bescimmre, daß der Dieb mir dem gestohlenen Gur auf dem Rücken zur Rechrsstätte, den ständig anwesenden Archonren geschleppt wurde (apagōgē = Wegführung); die Vertreter der Gemeinde übernehmen die Bestrafung. Hier wird die Selbsthilfe noch niche einfach verboren, sondern auf einen Weg gelenkt, der bei der Anerkennung eines öffenclichen Strafrechts ender. Der Angeklagte hat die Möglichkeit, anders als bei der reinen Selbsthilfe, die Rechtmäßigkeit seines Anspruchs zu vertreten; ein entscheidender Schritt auf die Rechtssicherheit hin. Aus dem Rächer ist schon der Kläger geworden. Auch bei der Entwicklung der Bußen ist diese Entwicklung erkennbar. Wenn der in seiner Ehre Geschädigte auf die Rache an der Person des Díebes, Ehebrechers usw. verzicbrete, konnte dieser sich mit einer Bußzahlung freikaufen (vgl. das germanische Wergeld und Entsprechends im römischen Zwölftafelgeserz); mit dem Auftreten des Geldes konnce es in Drachmen festgesetzt und sebr differenziert werden. Diese Festsetzung durch ein Gesetz ist allein schon eine Einschränkung der Selbscherrlichkeit des Rächers. Deuclich wird der Schritt zu einer Strafe im eigentlichen Sinn, wo die Buße zu einem Teil oder ganz an die Gemeinde geht (wo solche Strafen regelmäßig gezahlt werden, dürften sie in aller Regel den Gcbrauch von Münzgeld vorausse(zen). So teile das Solonische Gesetz über üble Nachrede die Buße zwischen Kläger und Staat; nicht so sicher datierbar ist der häufige Klagetyp 'wegen Mißhandlunge, wo die Strafsumme restlos an den Staat geht (und Popularklage herrscht, vgl. unten). Daß die Verletzung der körperlichen Unversehrtheit in Achen klar zum Bereich des öffentlichen Strafrechts gehört, verdient besondere Hervorhebung.

Zugleich ist hier allerdings ein Hinweis auf die Beschränktheit der öffentlichen Strafgewalt norwendig, um vor modernisierender Uberschätzung zu bewahren: es

10 Diesc sprichwörtlichen Ausdrücke stammen aus des Artikc - auf entwickclucem Niveau wandte man sich schaudernd von der Grausamkeit der frühen Geseugeber ab.

i Vgl. K. Latse, Beiữige zum gricch. Stralrecht. In: Hermes, Bd. 66 (1931), S. 270 f. (abgedruckt auch in: ders., Kl. Schrificn, München (968), 
stehen nur recht grobe Instrumente zur Verfügung, also vor allem die Buße (zu Solons Zeit sicher noch weit überwiegend nicht in Geld, sondern in Naruralien), die Ehr- und Friedlosigkeit bzw. die Verbannung und die Todesstrafe, gewöhnlich verbunden mir Gütereinzug. Es fehlt also so gut wie völlig die Gefängnisstrafe, außer als Uncersuchungshaft. Die geringe Differenzierung der Strafen wurden noch dadurch gesteigert, daß die Definition der Tatbestände rie die uns selbstverständliche Präzision erreichte (von Generalklauseln einmal abgesehen) und daß die Bestimmung des Strafmaßes weitgehend dem Ermessen der Richter überlassen blieb (vgl. den Prozeß gegen Sokrates wegen sGotrlosigkeit،). ${ }^{\text {2 }}$

Eine entscheidende Ausdehnung der persönlichen Freiheit und damit der Voraussetzung einer Freiheit des Willens (denn Sklaven unterstanden prinzipiell der unmittelbaren Getvalt ihres Herrn) bedeutete die berühmte und bereits erwähnte ,Lastenabschüttlungr durch Solon im Jahr $594 / 93$. Indern er die versklavten und verkaufren Bauern befreite und die Personalexekution für die Zukunft ausschloß, erhöhte sich die Zahl der dem Gesetz und der Gerichtshoheit unterworfenen Vollbürger erheblich (vermurlich mehr als die Hälfte der männlichen Bevölkerung Attikas). Solon selbst berichtet: Dem Edlen und Gemeinen schrieb ich gleiche Saczung,/gerechter Spruch gilt nun für jeden Streit ${ }^{1{ }^{13}}$

Für die wachsende Anerkennung der Autonomie der Person sprechen vor allem zwei Bereiche aus der solonischen Geserzgebung: aus dem sbürgerlichen Rechtr't das Testamentsgesezz; sodann das Instiru der Popularklage. Die Popularklage, auch später charakterisciscb für das atrische Recht, gestatter jedem (Voll-)Bürger, eine strafrechcliche (ebenso wie eine privatrecheliche) Klage einzuleiten (eine öffentliche Anklagebehörde hat es in Athen nicht gegeben). Während bei dem erratischen Block des Blutrechts deas Klagerecht bei der Gruppe der Blutsverwandten blieb, wurde also in allen übrigen Fällen von der Vorausserzung der Verwandtschaft abgesehen. Jeder einzelne Bürger sollte aus den alten Gruppenbeziehungen befreir und für das Recht verancworclich sein; die Scoßrichtung der Maßnahmen ist klar: "indem Solon ein Recht, das bisher in jedem Fall nur einer bestimmten Gruppe zustand, allen Bürgern einräumte, zerschnitz er ein Band, das diese Verbände innerhalb des Staates zusammengehalten und abgesondert hatce. Es war ein Versuch, die Solidaritär der Genossenschaften durch ein Gemeingefühl aller Bürger zu erseczen.«'s

${ }_{12} V_{g l}$. bes. E. Ruschenbusch, DIKASTERION PANTON KYRION. In: Historia, Bd. 6 (1957), S. $257-74$.

I) Fragment 24,18 Diebl, nach der Ubersetzung von $K$. Latte, Der Rechisgedanke im archaischen Griechenland (1946). In: ders., KI. Schriften, A. a. O., S. 24s. Latte bemerkı dazu: •Die Einführung geschniebener Gesetze bedeutet zunächst Gleichheit aller Vollbürger vor dern Geserz.... Isonomie, Gleichheit der Rechte, heißt das Schlagwort dieser Jahrhunderte. (Ebd.) Vgl. auch dic Verse des Euripides: ${ }^{-F e i n d s c l i g e r ~ i s t ~ d e m ~ S t a t e ~ n i c h e s ~ d e n n ~ d e r ~ T y r a n n . ~ / ~ Z u e r s t: ~ D o n ~ s i n d ~ G e s e r z e ~ n i c h t ~}$ gemeinsam Gut, / Gewale hat ciner, der besirzet das Gesetz/ Allein für sich - und dies ist nimmermehr gerecht " (wörnlich: gleich)./ wjedoch, wenn aufgezeichnet dic Gesetze sind, J Dann hat der Schwache und der Rciche gleich das Rechi,/ Berufen mögen sich dic Schwächeren darauf/Gen einen Mächriger, wenn er einen hat gekeränkt./ Und es obsicge des Großen der Geringre, hax er Recht. * Euripides, Die Flehenden, Verse 429 ff. Nach der Ubersetzung von F. SLoessl, Bd. 2, Zürich und Sturgar 1968).

14 Der Begriff ist nur mit Vorbehale anzuwenden, vgl. die Ausführungen weiter unten. Zum bürgerlichen Recht sind bei Solon zu rechnen: die furmilienrecbulichen Bestimmungen, die Regelung der Grunddienstbarkcien, der Bebauung und der Bepilanzung von Grundsrucksgrenzen, das Gesezz über Vercinsautonomie. (Nacb E. Ruschenbusch, PHONOS. Zum Reche Drakons und seiner Bedeunung lür das Werdco

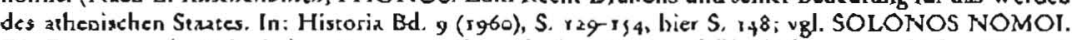
Die Fragmente des Solonischen Gesetzeswerkes mit einer Text-uod Uberlieferungsgeschichte. Hrsg. $E$. Rusclenbusch. Wiesbaden 1966, S. 25 f.)

15 Latte, Beiuräge zum griechischen Straf recht, a. a. O., S. 264 f. Lane weist darauf hin, daß dies auch die Absicht der Reformen des Kleishenes gewesen ist, auf dic in unserem Zusammenhang niche näher eirgegangen werden kann. (Kleisthenes machte das Territorialpriozip ansiclle der alten gentilizischen Eintcilung zur Grundlage des Büngerrechis und zerstöne so die Bundungen an die berkommlichen. viellach klasseumäBigen Gruppen). 
Historisch isc offensichtlich - das ließe sich auch an der frühbürgerlichen Geschichre des modernen Europas zeigen - die Emanzipation des bürgerlichen Individuums gebunden an die Entstehung einer sanksionsmächtigen öffentlichen Instanz, einer selbst nicht mehr unmittelbar von den Handlungszrvängen des Warenverkehrs abhängigen zentralen Gewalt, die nicht ohne weiteres allerdings ein politischer -Apparat in dem uns meist álzu selbstverseändlichen Sinn sein muß. Diese Aufgabe zumindesr muß jedoch geleistet werden, und sei es auch durch einen absoluten Fürsten, , Tyrannen', oder durch eine Körperschaft erwa nach Art einer Kaufmannsgilde oder selbst eine Stammesreligion (die für jüdische Kaufleute viel von dem verpflichtenden, ja zwingenden Charakcer jener Instanz übernehmen konnte). Dabei ist in keiner Weise an ein einseitiges Abhängigkeitsverhältnis zu denken, vielmehr an einen umfassenden Prozeß, in dem nur zeitweise einzelne Momente führend sein mögen. (In der athenischen Entwicklung ist übrigens schon früh die Unterscheidung von Rechtsetzung und Rechrsdurchsetzung erkennbar, wenngleich nicht in Gestak deutlich unterschiedener, ja gegeneinander verselbständigrer staatlicher Gewalten.) Im übrigen ist mit diesen Feststellungen keineswegs gesagt, daß der Staat der Arhener wesentlich durch diese rechtsstaatlichen. Aufgaben bestimme war.

Die Emanzipation des Individuums aus der traditionellen , Sippenhafrung und seine Anerkennung als Privateigentümer wird als Perspektive noch deutlicher erkennbar im Testamentsgeserz Solons. ${ }^{16}$ Für den Fall des Fehlens männlicher Nachkommen wird dem Erblasser hier Testierfreiheir zuerkannt, d.h. das Eigentum wird ein Stück weit aus seiner bedingungslosen Uncerwerfung unter den ökonomisch-religiös-verwandeschaftliclen Zusammenhang der Großfamilie befreit. Besonders bemerkenswert ist hier, daß das Tescament nach dem solonischen Gesecz bei Willensmängeln angetochten werden kann, z. B. bei Schwachsinn und bei Gewalteinwirkung. Damit sind schon zwei wesentliche Vorausseczungen für die Willensfreiheir der Person festgestellt (freilich charakteristisch unbestimmt, denn es werden keine Kriterien genannt, was zu endlosen Anfechrungsklagen geführt hat; überbaupt hat es im griechischen Recht nie eine gesetzliche Theorie der Willensmängel gegeben).

\section{Schranken der Entwicklung des griechischen Rechts}

Von verschiedenen Autoren werden weitere Zeugnisse für die «grundsätzliche Anerkennung des Privateigentums (daneben auch von Leben, Freiheit, Ehre usw.) angeführr ${ }^{17}$, z. B. die Unantastbarkeit des Hauses oder der seit dem frühen 7. Jahrhundert übliche Eid der Jahresbeamten, in dem die Anerkennung aller bestehenden Eigentumsverhältnisse proklamiert wird. Die verschiedenen $Z$ weige des athenischen Rechrs sind dabei nach den Kriterien des enrwickelten römischen Rechts untersucht worden, ein leicht zu Fehleinschätzungen verführendes Verfahren; es seien einige charakterisusche Titel genannt: Griechisches Privatrecht auf rechtsvergleichender Grundlage; Untersuchungen zur Geschichte des athenischen Strafrechts; Studien zum öffentlichen Recht Azhens ${ }^{\prime 8}$. In verschiedenen neueren Untersuchungen sind durch tiefer eindringende, z. T. durch die Ethnologie und die vergleichende Rechts-

16 Solon, Fragment 49, ya, nach der Ausgabe von Ruschenbusch, a. a. O.

1) $\mathrm{Vgl} . U$. Kabrstedt. Studien zum öffentlichen Rechi Athens. Tcil I: Staatsgebict und Staatsangeborige in Athen. Szungare 1934 (= Aalen 1969), S. 129 fí., hier S. 138.

18 E. Weif, Griechisches Privatrecht auf rechesvergleichender Grundlage, 1, (cinziger) Band: Algemcine Lehren. Leiprig 1923 (= Aalen r965); U. Kahrsedt, Studicn zum offentichen Rechr Athens. Teil I, 2. 2. O. Tcil Il: Untersuchungen zur Magistratur in Achen. Srutgart 1936 (= Aslen 1969); $E$. Ruschenbuscb, Untersuchungen zur Geschichte des athenischen Stralreches. Käln, Graz 1968. 
geschichte angeregte Interpretation allerdings mehr die Schrankea der Entwicklung des griechischen und auch des attischen Rechts herausgearbeiter worden. ${ }^{19}$ Gerade weil sich diese Arbeiten hauptsächlich auf die Rechtsentwicklung der klassischen und hellenistischen Zeit beziehen, sind sie interessant für die Schranken selbst der späteren Entwicklung des griechischen Rechts, die den Begriff des Privatrechrs selbst fragwürdig werden lassen (und daher auch den Begriff des Staatsrechts, ja überhaupr des Seazes, der Souveränitär und des Rechts beschränkc erscheinen lassen). Es soll dies im folgenden an der beschränkcen Encwicklung der Begriffe des Privateigentums und des Verrags gezeigt werden; die volle Emanzipation von gebundenen Formen des Eigentums zum Privateigentum ist ja die Voraussetzung für die Konstitucion einer bürgerlichen Gesellschaft im modernen Sinn des Wortes.

Zunächst fälic bei genauerer Betrachtung auf, daß es keinen Begriff für Privateigentum gibt (und daher auch keine Unterscheidung zwischen Eigentum und Besitz), durchaus im Unterschied vom entwickelten römischen Recht (dominium, dominium ex jure Quiritium). Das Won usía meint sowohl, Vermögen, Habe, Eigentume wie Persönlichkeit, Wesen . Ausdrücke, die jemand gehören bezeichnen, beziehen sich wesentich auf die tarsächliche physische Kontrolle und auf einen gewissermaBen auch psychischen Anspruch auf eine Sache. Am nächsten im Deutschen kommt noch das Wort ,Haben oder das schwäbische , mei eigen', 'mei Sach $\times$ Zu erinnem ist übrigens auch daran, daß die erlaubre Selbsthilfe sich auch für uns auf das eigene Selbst als körperliche Person wie auf körperlich vorhandenen Besitz bezieht. ${ }^{20}$ Auch etwa das Wort chrêmata, oft übersetzt als ,Geldmittelk, ist vieldeutig; sein Stamun verweist auf den Nutzen für den Gebrauch, auf das Bedürfnis; es kann dann die Masse nützlicher Gegenstände sein, das Vermögen, der Besizz, die Geldsumme, ja die Schulden oder die Waren."

Wie vergleichsweise wenig die Vorstellung des Privateigentums im griechischen Recht entwickelt ist, zeigr sich auch deurlich an den Rechtsmirteln zur Wiedererlangung beeinträchtigten oder verlorenen Eigentums. Immer ist es der Besitzer, der gezwungen isr, seine Ansprüche zu rechufertigen; durch Klage konnte das relativ bessere Recht, dagegen nicht ein absolutes und ausschließliches Eigentumsrecht festgestells werden (so war immer möglich, daß ein Dritter mit noch besserem Rechr auf(rat). Nur in dieser Form wird im Urteil zum Eigentumsrecht Scellung genommen, also keine Anerkennung und Bestärigung eines subjekciven Rechrs ausgesprochen; das Urteil gilt vielmehr noch als Abschluß eires Streits. Man findet auch nicht den Begriff der Forderung im Sinne des römischen Rechts, Und die Wiederherstellung des verlorenen >Vermögens، war eine Wiederaneignung im wörtlichen Sinne: es gab keine direkte staatliche Hilfe bei der Vollstreckung, sondern der Obsiegende erhielt durch das Urteil nur ein Zugriffsecht, er mußte sein Recht selbst durchsetzen, z. B. durch persönliche und gewaltsame Wegnahme vom Vorbesitzer oder durch Zugriff auf den Schuldner. Erst wenn dieser das seinerseits mir Gewalt verhinderte, wurde ein besonderer Klagetyp möglich (der eine Buße in Höhe des Streitwerts an den Staat vorsah); darauf konnte erneut zur Selbsthilfe geschritten

19 Vgl. zum folgenden insbes.: F. Pringsheim, The Greek Law of Sale, Wcimar rgso; H. f. Wolff, Die Grundlagen des griechischen Veturagsreches. In: Zeitschrift der Savigny-Suftung für Rechesgeschichte, Rom. Abr., Bd. 74 (1957), S. 26-72; Gernet, Uber den Begriff des Urteils .... a. a. O.; ). W. Jones, The Law and Legal Theory of the Grecks. $A_{n}$ Introduccion, Oxford. 1956, Kapitcl XI.

$20 \mathrm{Vgl}$. $227,859 \mathrm{BGB} ; \$ 53 \mathrm{SrGB}$.

21 Vgl. H. G. Liddell, R. Scolt, H.S. Jones, A Greek-English Lexixon, Oxford 1940, Unrer chrêma. Dicsen Doppelscharakter har auch unsere Sprache viclfach noch bewabre, womit die Schwicrigkeit angedcuret ist, alles rücksichtslos vom Siandpunkt des Werns aus zu betracheen. 
werden. Diese 2 Eigentumsklages war wesentlich "a form of execution, of enforcing rather than establishing rights, a means of realizing the power of self-help which early law regarded as bound up with thac sphere of power which was involved in its concept of property $\mathrm{K}^{22}$. Es kommr in diesem Verfahren sehr deutlich die konkrer-individuelle Beziehung zum Ausdruck, die der ,Eigenrümerı noch zu seiner Sacher"s har. Diese Sache gait als Teil seiner Persönlichkeit ${ }^{4}$, genauer: als Teil seiner gesellschaftlichen Existenz in der bescimmen Funktion der Lebenerhaltung im Gemeinwesen der Polis. Sein, Vermögen ist ein Teil seines persönlichen Herrschaftsbereichs innerhalb dieser spezifischen Gesellschaftsform der Polis, nämlich in dem besonderen Sinn, den dieser Bereich für den freien Achener hat: als Herr eines Oikos, einer Hauswirtschaft mit Grund und Boden, mit Haus und den notwendigen Werkzeugen und Gebrauchsdingen, mit ihm unterzanen Menschen (darunter Sklaven, in eingeschränkter Weise auch Frauen und Kinder; schließlich auch Tieren)."s

Diese konkrete Beziehung zum Eigentum, die noch weit entfernt ist vom ius utendi et abutendi des römischen Rechts und die prinzipiell auf die Aufgaben als Achener, als Polítes, Vollbürger der Polis Athen bezogen bleibr, drückt sich auch darin aus, daß der für das römische Reche zentrale Begriff der obligatio, der Vorausseczung des Schuldrechts, im griechischen Recht nicht ausgebildet worden ist, daß die Kategorie des Vertrags vergleichsweise unenrwickelt geblieben ist. Zwar war der Vertrag, der im allgemeinen mündlich vor Zeugen geschlossen wurde, durchaus bekannt, sowohl im alläglichen Kredit- und Darlehensverkehr wie in den auswärtigen Beziehungen;

22 Jones. The Law and Legal Theory ..., a. a. O., S. 204.

2) Am deuschen Wor Sache läß sich noch dic Enrwicklung von der konkreten zur abstratien Beziehung illuscrieren. Sache (bucinisch res) ist der juristische Terminus für Ware bzw. Privateigentum. Zu ihr hat man als Person, d. h. als Privarcigennümer ein sachliches. Verhälenis, kann sie ohne Schmerz verkaufen. Im schwäbischen mei Sach hingegen steckt noch deutlich die konkrete Beziehung: gemeint wird damit etwa meinc Argelegenheiten, Aufgaben, sdie nürzlichen Dinge hür cine besrimmı Tärigkeite, überhaupe swas mir zugchörte. Man ist dabei immer auf eirc konkrete Akrion bezogen, 2 . B.: i han mei Sach zammar sagr eine Frau, wenn sie vor dem Baden Handiücher, Seife usw. zusammengelegr bar. Das hochsprachliche $\mathbb{W} \circ \mathrm{r}$ ssachliche wird im Schwäbischen nichı gebrauche. Andercrseits kann man schr wohl ssei Sach zammahaleax, räolich sparsam, wenn nicht geizig - der Scbazzbildner als Frühform des akkumulierenden Kapicalisen ist dor niche unbekannt. (Vgl. zu dieser bürgerlichen Figur die Hinweise in meiner Untersuchung: Geld und Geist, S. 8 I f. mit Anmerkung s8; zum Schwäbischen vol. Th. Troll, Deuschland, deine Schwaben. Hamburg 1967, bes. Kapitel 9: Das Sach.) Solehe Beobachrungen lassen sich an vielen Dialekıausdrücken machen. Dialekrc ragen gewissermaßen auf der vorkapitalisuischen Vorstufe in unsere Gesellschaft hinein. Ihre vorbürgerlichen, häufig bäucrlichen Sprachformen drücken viel stärker die dirckıen, remotionalen (wic der disıanzgewohnte Bürger sagt) Beziehungen zu Menschen und übriger Umweit aus. Hochsprachen dagegen sind niche nur Mirrel bürgerlich-nacionaler Vercinheiblichung, sondern Sozialisationsmirtel für küh-disuanicrecs Verhalecn, Abstraktion und Rücksicheslosigkeit gegenuber cigenen und fremden, Gefühlen. (die Einübung in die klassische larcinische Hochsprache hat jahrhunderedang diese Aufgabe crï̈llt; heutc sind die Hochspracben entoprechend slogische geprägt; nicht zufälig haben dic absoluciscischen Fürsten Liecinschulen errichect, um dic juristische Schulung der Diencr des Allgemeinen vorzubereiten). In der En(wricklung solcher sarchlichen', abstrakien Beziehungen stecke die Voraussetzung von Wisserschati überhaupe. Eine Enrwick. lung. welche besonders dor an schmerzhafe Schranken stößs, wo die distanzier veräußierze Sache unablösbarer Teil der eigenen Existenz, ఒ. B. das eigenc Arbeitsvermogen ist.

24 Im vorrevolurionären China hatte der Verkäufer zeidobens das Reche, zu bestimmten Festen seiner verkaufen Sache Seufzerbillertse anzuheficn und damir seincn Schmerz über die Veräußenung auszudrücken, die Veräußcrunga also eines Teils seines Selbst. Vgl. M. Masss, Die Gabe (1924). Frankfurt I968, S. Is5.

2s Ubrigens ist noch bei John Locke der Begriff Property ähnlich schillernd; er meinz sowoh! Herrschaft/ Eigentum über den cigenen Körper wie ibocr die Gebrauchsdinge der Umwels, die durch eigenc Arbei der naeürliehen Umwelt abgewonoen sind. Locke läße frejilich den Begriff sich enrwickeln, so daß einerseits die Macht über den eigenen Körper als Eigentum an dessen Arbeirsvermögen erscheint, andererscius fleiRig-rationale Menschen durch Aufhäufung erarbentever Gebrauchadinge sich die zeirweise Veriügung uber die Arbeitskralt anderer eintauschen können, und daher Akkumulation möglich wird. Vgl. Locke, Two Treacises of Government، Second Treatise. Hg. P. Lsslcte, Cambridgc 1970'. 
auch wurde die Nocwendigkeit einer öffentlichen Sankcion klar gesehen. ${ }^{16}$ Jedoch bedurfe er gnundsätzlich einer realen< Basis, d. h. er wurde zu einem bestimmten Zweck abgeschiossen, dessen Nichterfüllung die Haftung des Schuldners begründece. Nur diese sSchädigungı konnte eine Klage begnünden, die einen vergeltenden Zugriff ermöglicben sollte. Die bindende Wirkung konnte also nicht direke aus dem Konsens, dem im Vertragsschiuß bekundeten Zusammenhang mehrerer Willenserklärungen abgeleicet werden, wie es der Konsensualvertrag des römiscben Rechts vorsieht. Die Entrtehung der Verpflichtung gilt als Wirkung der Ubberlassung des konkreten Gutes, z. B. eines Grundscuicks, bzw. als Wirkung der Schädigung durch Nichtherausgabe usw. (Die Dinge treten noch selbst in Erscheinung, weil sie als Waren noch richt voll enrwickelt sind; deshalb sind ihre Besiczer auch nicht in der Lage bzw. gezwungen, sie losgelöst von ihren konkreten Interessen und Bedürifnissen bloß als Träger von Wert, als Waren bzw. als Kapical zu betrachten. Die , Herten dieser Dinge betracheen einander noch nicht als bloße Repräsentanten von Waren und Geld, als autonome Privareigenrümer, deren Willen nur Ausdruck der Waren und ihrer mehr oder weniger verselbständigten Bewegung sind. Wo dies aber doch schon geschieht (z. B. im Wucherkapital), wird es abgewehr und abgelehnt (vgl. die Figur des Geizhalses)" ${ }^{2}$ - die Hefrigkeit der Ablehnung mag immerhin bezeugen, wie sehr sich die Autonomie der Wertbewegung bereits bemerkbar machce. Hier ist allerdings die Frage anzufügen, ob sich die Mitglieder selbst einer entwickelten kapizalistischen Gesellschaft rein als autonome Privateigentümer, als bloße Repräsentanten von Waren und Geld, bzw. der in diesen Formen sich äußernden Bewegung des Werts betrachcen und behandeln können. Sie sind diese Rechtspersonen bzw. Charakcermasken doch immer nur sorveic sie ihre unmittelbaren Bedürfnisse - wie immer gesellschaftliche verminelt diese sind - zurückdrängen, sich moralisch domestizieren auf jenes Allgemeine hin, daß ihre Gesellschatclichkeit ist (und das ihnen äußerlich als Staat gegenübertritt, ob nun durch ihre freiwillige Ubereinkunft begründer oder als unvermeidlicher Not- und Verstandsstaat).)

26 Schon Herodor (Mitte des s. Jahrhunderts) bemerkt: Ohne zwingende Sicherung werden Vcrträge nicht sicher bleiben *, bezogen freilich au! Verträge zwischen Fürsten bzw. Sraaten (Hesodor, Historiae, I, 74) In den überlieferten Gerichtsreden (vor all cm im 4. Jahrbundert) ist es eine stehende Redewendung, daß Verragsparteien sich an ihre Versprechen zu halten haben, und Avistotcles hält gesellschafilichen Verkehr ohne sereitschlichrende Gerichte fur ausgeschlossen; vgl. Jones. The Law and Legal Theory ...., 2. a. O., S. 216

27 Als einziges Beispicl aus zahllosen dieser Arr (ubrigens auch unserer Zeit, manchmal reakrionär mißbrauche) einige Verse aus der Antigone des Sopbokles (s. Jahrhundert v. Chr.; Verse 295 H.):

Denn unser allem, was in Brauch ist unter dey Menschen,

Eraruchs so schlimen niches wie das Geld! Dieses zerstör

Sclbsı Së̀dre, dieses treibı Männer von der Häusem,

Dies lehre und es verkehn den nechten Sinn

Der Menschen, üblem Tun sich zuzuwenden.

Wie man zu allem fähig wird, zeigr es den Mcnschen

Und jede An Gorklosigkeit zu wissen. -

Man sah also nur die zersetzende Seite, die Zersiörung der aleen Ordnung, und konnte auch sehwer erwas anderes sehen, denn die Endalrung der produktiven Kräfre der menschlieben Arbcit war nur ganz peripher von dem Vermchrungsdrang des Geldkapicals ergriffen; cine Entfaltung, welche immer auch eine Entfaltung der Destrukcionskü̈fe gewesen ist und die heuce spürbar und un widerruflich die innere wie die äußere Nacur zu zerstören drohz. Wescntlicher Kern dieser Entralung ist sichet die Erzcugung des abstrakten Reichtums, die Bereicherung als Selbstzweck; dieser endlose Prozeß ist jedenfalls nicht erst mir dem Kapical im Verhälmis zur Lohnarbeir geseczu, sondern bereits mit dem Geld in seiner drinen Formbesammeheic (vgl. zur Bedeutung dieses Bcgrifts die Hinweise in meiner Arbcit "Geld und Geista, a. 1. O.). Andererseirs hat sich der Automausmus dieser endlosen Bcwogung längst eigene Strukturen außerhalb des Kapitalverhälenisses im engeren Sinn geschaffen; erwa in der Sunkiur wissenschafilicher Forschung, die nicht in erster Linie wegen ihrer Finanzicrung durch zahlungskeäftige Interesserneen destrukuv sein kann: in dem Typus des Denkens eines Galilei steckt der Möglichkcit nach die Atombombe, wie es Brecht ausgedrückt hat. Die Rede von der, wissenschafilicb-technischen Revolution als eigenständigem Antrieb hat insofern recht, aul bewolitlose Weise. 
Wie sehr der Gläubiger erwa eine konkrete Beziehung zu seiner Sache hat, zeigt sich an der Vorssellung, daß das Darlehen nicht in das Eigentum des Schuldners übergehr, diesem zur freien Verfügung (und das heißt zur Vermehrung mir beliebigen Mitreln) übereignet ist, sondern Eigentum des Gläubigers bleibr (unjuristisch auch noch bei uns, wenn jemand sein Geld، zurückfordert, das er jemand geliehen hat). "Wo immer in den astischen Reden von Darlehen gesprochen wird, da hören wir den Darlehensgeber sagen, daß der Entleiher sein Geld habe und ihn dadurch, daß er nicht zahle, um sein Geld beraube (aposterein). Von der scharfen Abgrenzung des entwickelcen römischen Rechts, bei welcher das als muturum oder despositum irregulare kveditierte Geld ins Eigentum des Schuldners übergeht, der dann aufgrund der angeknüpften Schuldbeziebung auf Rückgabe hafter, ist niches zu bemerken. ${ }^{28}$ Vielmehr gibr der Gläubiger das Darlehen, auch wenn es Geldform hat, zu einem bestimmıen Zweck, nichi zur beliebigen, allerdings zinstragenden Verwendung; die Verlerzung dieses Zwecks, die Scbädigung durch Nichtausfühnung der verfügten und vom Versügungsgegner angenommenen Bedingungen, nichr der einfache Bruch des gegebenen Versprechens, begründec das Reche zum gerichtlicben Vorgehen. In den Gerichcsreden hebr Demosthenes selbst im Falle des Seedarlehens die reale Natur des vom Beklagten streiwillig im Empfang genommenen und dann weggenommenen Talents hervor. Beruhte die Beziehung nur auf ,Glauben<, wäre weder Pfand- noch Bürgschaft geleister, so wäre eine Klage strenggenommen nicht möglich. ${ }^{29}$

Der griechische Vertrag kennt also noch nicbt die volle Anerkennung des freien Willens der Vertragschließenden, der abstrakeen Rechtspersonen, im Sinne des römischen Rechrs; er ist nicht wirklich gegenseirig, sondern trägt noch deutlich Spuren der Ungleichheit der Beteiligten. Wenn also der Vertrag wesentlich »Zweckverfügung" (Wolff) blieb, so gilt das Entsprechende für die Karegorie des Ureils und des Privateigentums: das Eigentum, das als subjektives Recht um seiner selbst willen öffenclichen Schurz genießst und im Urteil explizit bescätige wird, isc dem griechischen Recht fremd geblieben. In diesem Sinn gibr es daher kein Privatrecht in der antiken griechischen Polis. Dies alles bezieht sich im übrigen ohnehin nur auf die männlichen Vollbürger; daß deren Frauen und Kinder und vor allem deren Sklaven nicht oder nur sehr beschränkt rechtsfähig waren, ist bekannt. ${ }^{\text {jo }}$

28 Wolff, Die Grundlagen ..., a. ., O., S. 9081 .

29 Tarsächlich gibt es nach Anstoseles (Njkomacbische Ethik, IX, 1, S. 1164 b 13 f.) in einigen Poleis keine Möglichkcit der Klage für den Gläubiger von Gelddarlehen; entsprechend woltte Plason, daß im idealen Staat dic meisten ofreiwilligen Rechrsgescbäfrc auf Gefahr des Gläubigers crílgen sollten, Normalfall sollte das Bargcschăfz scin (Platon, Seant, Buch VIII, S. $36^{6}$ a, b). Die Verteagsvorstellung blieb jm griechischen Rochr immer im Umkreis des Barkaufs stehen; obwohl Kreditkaul und Aozahluogskaul spärestens seir der klassischen Zeir eine sehr wichrige Rolle gespielt haben, sind sie nicht in der Form des Verrags ausgedrück! worden. Vgl. zusammenfassend Jones, The Law and Legal Theory ...., a. a. O., S. 228: *... there is good ground for supposing that Greek law, like early Roman and English law, considered salc as essentially an exchange of land or goods for money, a cash transaction giving no right to enforce payment on the one side or delivery on the other. It was a two-sided affar; but therc could be no question of any outstanding obligations arising from the simple lace of agreement in law the salc was complete or it was nothing and it was nor complete unul the price had been paid ..... Dic wichugste Untersuchung zur Frage des Barkaufs und seiner rechlichen Ausdrucksiom: Rringsheim. The Greek Law of Sale, a. a. O.

30 Allendings ist die Vorstellung völliger Rechtslogiskeit der Sklaven niche richrig, besonders wenn über den Bereich des Privatrechus hinausgegangen und das (öffendiche) Surafrechr und das Sakralrecht berürksichtigg wird. Uber dic geminderie Rechessiellung der ansässigen und anerkannicn Auslinder (Meiöken) und der voribergehend anwesenden Auslinder (Xenoi) kann genaveres nur gesaze werden, wenn das Handelsrechr im engeren Sinn und der durch Verreäge mit anderen Sudrseanten bewirkic Rechrsschurz benicksichuig wird; hier ist cine alimähliche Annäherung an den Status des Vollbürgers lestzustellen, allerdings nicht hinsichulich der politischen Rechte. 
Abscbließend möchte ich zu den Wirkungen kornmen, die nach meiner Einschätzung von Wesels Aufsatz, zumindest wenn er obne zusätzliche Erläuterungen aus dem Gesamtzusammenhang einer Vorlesung herausgenommen ist, in der gegenwärtigen Situation einer tiefgreifenden , Kulurkrise ausgehen können. Einer Kulturkrise, die durch die Entstehung einer ökologischen und alternativen Bewegung am deurlichsten bezeichnec werden kann und die auch und besonders die Linke ergriffen hat. Nach meiner Auffassung ist eine solche Krise unumgänglich, wenn nicht Kultur überhaupt untergehen soll. Alle Konzeptionen und Theorien, die seit dem Auftreten des Kapitalismus in Europa entstanden sind, bedürfen einer radikalen Uberprüfung, eingeschlossen darin die Marxschen Auffassungen. In diesen Jahrzehnten einer menschheirsgeschichtlichen Krisenphase mir der Möglichkeit eines katastrophalen Ausgangs ist es nocwendig, Ursprung und Geschichie dieser Menschheic zu erinnern und andere Gesellschaftsformen im Hinblick auf ihre und unsere Ubberlebensfähigkeir kennenzulernen. Gerade die Beschäfrigung mir dem 'ganz Anderen kann sehr fruchrbar sein für eine durch kulurellen Schock distanzierte Wahrnehmung und Kritik der eigenen Gesellschaft. Aber diese Distanzienung kann nur sinnvoll sein und fruchtbar werden, wenn sie als intellektuelle und durchaus auch emocionale Veranstaltung verstanden wird, welche in ihren Erfahrungsformen, Interessen und vor allem alläglichen Lebensvoraussetzungen von den bestehenden gesellschaftlichen Verhältnissen ausgeht und in sie zurückgeht. So sehr man es bedauern mag: es gibr keinen Abschied aus dieser Gesellschafr, oyeder für Gruppen noch für Einzelne. Solche Abschiedsversuche, solche abstrakc-utopische Sehnsucht kennen wir historisch aus bestimmten Varianten der Romantik, auch der politischen Romantik dieses Jahrhunderts. Derartige Versuche haben durch ihre unvermittelre Identilikation mit dem ganz Anderen und sicher meist gegen ihre bewußte Intention zur Erhaltung und Festigung der bestehenden, für Veränderung oder Umwälzung überfälligen Verhälınisse beigetragen. Gerade das abstrakt-utopische Verhalten ist in der Gefahr, aus dem Bestehenden einfach auszusteigen, dieses dadurch tatsächlich aber nur zu bestärigen; durch reine Verweigerung und/oder durch unbewußte Indienststellung. Es gibc kein einfaches Zurück. Jede Lehre, die wir aus besseren Formen des Lebens in anderen Gesellschaften ziehen können, bedarf einer sehs reflektierten Umsetzung auf die tatsächlichen und durch keinen Willensakr beiseitezuschiebenden Verhälnisse unserer Gesellschaft (einmal abgesehen von den entgegenstehenden Machtverhältrissen und Interessen). Und das trifft auch dann zu, wenn diese Gesellschaft insgesamt verändert werden muß. Im übrigen gilr, daß bestimmte Seiten der bürgerlichen Gesellschaft Errungenschaften auch für die heurige Situation sind und entschieden festgehalten werden sollten.

Im folgenden möchte ich an wenigen Punkten zeigen, wie solche Abschiedsversuche sich an einzelne von Wesels Argumentationen anhängen können. Weil sein Aufsarz nur von der frïben Entstehung des Staates und des Rechts handelt, sritt das Moment direkter Unterdrückung durch Eroberer stark in das Blickfeld. Im Anschluß an Meyer-Fortes und Diamond führ er aus, daß Kephaliär, Herrschaft, und damir Recht, gegen die alte Ordnung entstehe, welche durch Freiheit, Gleichheit und Brüderlichkeic gekennzeichnet sei und den kollektiven Konsens aller durch Gespräch und Schlichrung erreiche, als sich selbst regulierende Anarchie. Physische Gewalr, in den Jäger- und segmentären Gesellschaften nur vorhanden als individuelle, ungeordnete und von innen heraus durch einigenden Konsens überwunden, werde in den kephalen Gesellschaten zur herrschaftlichen, apparativen Gewalt

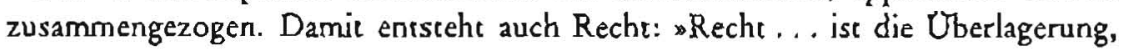


Absorption und Zerstörung von Gewohnheir durch zencrale Herrschaft, die es zunehmend und bewrßt als Instrumentarium von außen einsetzt, ist steuerbar, verfügbar und verbunden mic physischer Sanktion, also apparative Gewalt, während Gewohnheit das kollektive Gespräch war. a ${ }^{31}$ Die Rechrsprechung ist, nach der zwangsausübenden Gewalt, verkörpert in verselbständigter milizärischer Machr (z. B. einer Leibgarde), wesentliches Kennzeichen von Herrschaft. Daher hält Wesel die Theorie von der Ensstehung des Staates (und damit des Rechts) durch Eroberung für immer noch die sicherste, trotz einiger Einwände; gegen die Theorie von Engels (bzw. Morgan), nach der das Privateigentum der Motor dieser Entwicklung gewesen sei. Vereinfacht: Gewalc, Sraar, Herrschaft, Reche sind fast Synonyma und stehen gegen Friedlichkeis, Anarchie, Gleichheit, Freiheit, Konflikulösung durch Konsens, Brüderlichkeit. Wohlgemerkt: Wesels hier stark zusammengezogene Aussage (aber wie rezipieren Leser Texte?!) bezieht sich auf frühe Gesellschaften. Aber sie fügt sich bequem ein in eine dualisrische, das Moment der physischen Gewalt verabsolutierende Vorstellung vom bürgerlichen Staat heute, die in der Linken nicht so ganz ausgestorben ist. Doch das bürgerliche Recht und der Staat a's Recbrstaat sind gerade nicht Institurionen direkter Herrschaft. Bürgerliche Herrschaft ist verhüllte und in bestimmter Weise auch ambivalente Herrschaft. Indem der Staar als Rechestaat Freiheit, Gleichheir und Eigentum sichert, sichert er zugleich das Recht des Kapitaleigentums auf das volle im Produktionsprozeß erzeugre Produkr. Er sichert aber auch das Reche des Arbeiters als Eigencümer von Arbeitskraft; und weiter bierer die offensive Interprecation der Freiheits- und besonders der Gleichheitsrechte eine Einbruchsstelle in die durch das Recht zunächst eindeutig gesicherte bürgerliche Klassenherrschaft (vgl. allgerneines Wahlrecht, Mitbestimmung).

Auch an einem anderen Punkr schürzr sich Wesel $\mathrm{m}$. E. nichr gegen problemarische Interpretationen. Wiederholt hebr er bei Jäger- wie auch segmentären Gesellschaft die Konfliktregelung durch Diskussion und Verhandlung hervor, erkennt er ihnen das Prädikat 'Freiheir, Gleichheir, Brüderlichkeir zu. Diese Formulierungen erwekken unweigerlich Assoziationen an bürgerliche Ideale und Normen. Sicher sind einige dieser Ideale im Rückgriff auf vergangene, als brüderlich, friedlich usw. erinnerze Zustände formuliert worden, und sicher haben sie von Anfang an ein über die bürgerlichen Verhälenisse überschießendes Moment enthalten. Aber ihre Bedeutung innerhalb der bürgerlichen Gesellschaft ist überwiegend eine ganz andere als in vorbürgerlichen Gesellischaften. Sie sezzen die Anerkennung des Individuums voraus, oder sie fordern diese Anerkennung erst, über die bürgerlichen Schranken hinaus. Das isc erwas sehr anderes als die Gleichheit oder die Freiheir in akephalen Gesellschaften. Denn hier ist der Einzelne erwas ganz anderes als das Individuum in den bürgerlichen Gesellschaften (selbst als der einzelne der Unterschicht). Die Mitglieder solcher Gesellschaften sind aufs stärkste gebunden in hergebrachte Regeln des Verhaltens, mit sehr geringen Abweichungsmöglichkeiten für den cinzelnen, Wesel erwähnt selbst die festen Regeln, die bei der Jagd, bei der Beuteverteilung, bei der Arbeitsceilung zwischen Männern und Frauen, auch bei Heirats- und Sexualverhalten und bei Törungen bestehen. Isr es richtig, diese Regeln völlig vom Begriff der Herrschafr loszulösen? Erwa weil sie noch bloß Custom, nicht Law sind? Demnach einzelnen oder Gruppen nicht zur herrschaftlichen Disposition stehen? Auch wenn dies so ist, so härte doch deutlich gemacht werden müssen, wie sehr solche Cusroms für unsere Wahrnehmung alle übermächtig und geradezu erbarmungslos unentrinnbar beherrschen. Es könnten sonst sehr falsche Hoffnungen genährt werden, welche - so verständlich das auch im Einzelfall und so

31 Vgl. gegen Ende des Abschnitrs oW 25 ist Reche? $\propto$ 
sinnvoll das als Lemphase sein mag - das Bestehende durch scheinbare Sezession bloß affirmativ bestätigen und im übrigen für den einzelnen im Scheireen enden können"s.

"Anm. der Redaktion: Uwe Wesel wisd in cinem dor nächsten Heftc ancworten.

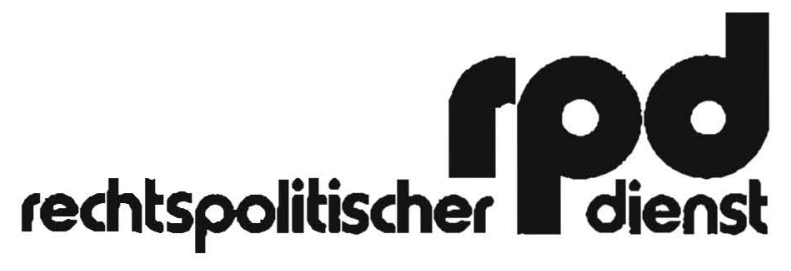

berichtet jede Woche aktuell aus Bundestag und Bundesrat, informiert über den neuesten Stand der Gesetzgebung, über wichtige Reformvorhaben und Novellierungsvorschläge, berichtet über rechtspolitisch interessante Gerichtsund Verwaltungsentscheidungen, über neue Entwicklungen im internationalen Recht, liefert die notwendigen Hintergrundinformationen und ermöglicht einen schnellen Überblick über die verschiedenen rechtspolitischen Positionen.

Auf Wunsch senden wir Ihnen den wrechtspolitischen dienst " kostenlos zur Probe. Rufen Sie inn noch heute bei uns ab.

\section{pressebüro ralf theurer}

